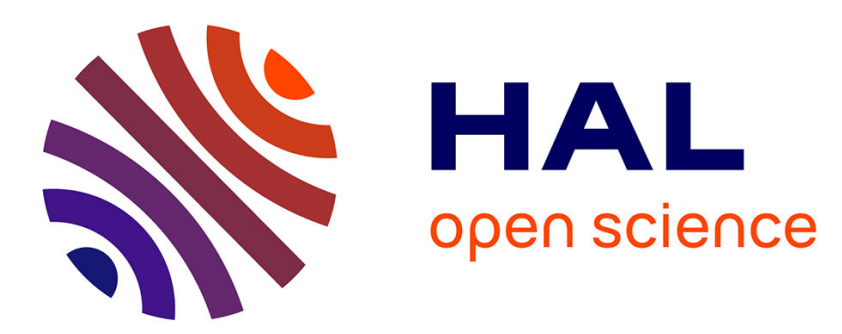

\title{
Control Solution for a Cable-Driven Parallel Robot with Highly Variable Payload
}

\author{
Etienne Picard, Stéphane Caro, Franck Plestan, Fabien Claveau
}

\section{To cite this version:}

Etienne Picard, Stéphane Caro, Franck Plestan, Fabien Claveau. Control Solution for a Cable-Driven Parallel Robot with Highly Variable Payload. ASME 2018 International Design Engineering Technical Conferences \& Computers and Information in Engineering Conference IDETC/CIE 2018, Aug 2018, Quebec city, Canada. pp.1429-1436, 10.1115/DETC2018-85304 . hal-01863730

\section{HAL Id: hal-01863730 https://hal.science/hal-01863730}

Submitted on 29 Aug 2018

HAL is a multi-disciplinary open access archive for the deposit and dissemination of scientific research documents, whether they are published or not. The documents may come from teaching and research institutions in France or abroad, or from public or private research centers.
L'archive ouverte pluridisciplinaire HAL, est destinée au dépôt et à la diffusion de documents scientifiques de niveau recherche, publiés ou non, émanant des établissements d'enseignement et de recherche français ou étrangers, des laboratoires publics ou privés. 


\title{
DETC2018-85304
}

\section{CONTROL SOLUTION FOR A CABLE-DRIVEN PARALLEL ROBOT WITH HIGHLY VARIABLE PAYLOAD}

\author{
Etienne Picard ${ }^{1,2}$, Stéphane Caro ${ }^{2,3 *}$, Franck Plestan ${ }^{2,4}$, Fabien Claveau ${ }^{2,5}$ \\ ${ }^{1}$ IRT Jules Vernes, Bouguenais, 44340, France \\ 2 Laboratoire des Sciences du Numérique de Nantes (LS2N), UMR CNRS 6004, Nantes, 44300, France \\ ${ }^{3}$ Centre National de la Recherche Scientifique (CNRS), Nantes, 44321, France \\ ${ }^{4}$ École Centrale de Nantes, Nantes, 44321 France \\ 5 IMT-Atlantique (Site de Nantes), Nantes Cedex 3, 44307, France \\ Emails: etienne.picard@irt-jules-verne.fr, stephane.caro@ls2n.fr, \\ franck.plestan@ec-nantes.fr, fabien.claveau@imt-atlantique.fr
}

\section{ABSTRACT}

This paper deals with the design of a robust control scheme for a suspended Cable-Driven Parallel Robot (CDPR), composed of eight cables and a moving platform (MP), suitable for pick-and-place operations of heterogeneous objects with different shapes, sizes and masses, up to a total load of $700 \mathrm{~kg}$. Dynamometers measure the force applied by each cable onto the moving-platform and are used to assess the payload mass at any time. In the proposed control solution, each motor of the CDPR is directly driven by a PD torque controller, which takes benefit of the real-time payload estimation in a feedforward term. In order to evaluate its performance, experiments on a typical pick and place trajectory are realized for different payloads. As a result, three control schemes: (i) a Proportional-Derivative (PD) torque controller; (ii) a PD controller with compensation of the MP mass only and (iii) a PD controller with real-time mass estimation and compensation are experimentally compared with respect to their positioning accuracy. It turns out that a good estimation of the payload is obtained in real-time thanks to the dynamometers. Moreover, the higher the payload mass, the more accurate the proposed controller with respect to its two counterparts.

Keywords : cable-driven parallel robots, modeling, robust control, payload estimation

\section{NOMENCLATURE}

$m \quad$ Number of cables

$\mathbf{l}_{i} \quad i^{\text {th }}$ cable vector

$\mathbf{u}_{i} \quad i^{\text {th }}$ cable unit vector

$A_{i} \quad i^{t h}$ cable exit point on the pulley

$B_{i} \quad i^{\text {th }}$ cable anchor point on the platform

$P \quad$ Geometric center of the platform

$\mathscr{F}_{b} \quad$ Frame attached to the base of origin $O$

$\mathscr{F}_{p} \quad$ Frame attached to the platform of origin $P$

${ }^{b} \mathbf{a}_{i} \quad$ Vector pointing from $O$ to $A_{i}$ expressed in $\mathscr{F}_{b}$

${ }^{p} \mathbf{b}_{\mathbf{i}} \quad$ Vector pointing from $P$ to $B_{i}$ expressed in $\mathscr{F}_{p}$

${ }^{b} \mathbf{R}_{p} \quad$ Rotation matrix from $\mathscr{F}_{b}$ to $\mathscr{F}_{p}$

W Wrench matrix of the robot

J Kinematic Jacobian matrix of the robot

t Cable tensions vector

$G \quad$ Center of mass of the platform and additional load

$\mathbf{w}_{g} \quad$ Wrench applied to the platform due to gravity

$m_{p} \quad$ Mass of platform and load to compensate

MP Mobile platform of mass $366 \mathrm{~kg}$

$\mathrm{M}_{1}, \mathrm{M}_{2} \quad$ Metal plates of mass equal to $122 \mathrm{~kg}$ and $249 \mathrm{~kg}$

PD Proportional-Derivative torque controller

PDFF PD controller with feedforward term (FF)

for MP mass compensation

PDFFRT PD controller with feedforward term for real-time (RT) mass estimation and compensation

*Address all correspondence to this author. 


\section{INTRODUCTION}

Cable-Driven Parallel Robots (CDPRs) form a particular class of parallel robots whose moving platform is connected to a fixed base frame by cables, as illustrated in Fig. 1. The cables are coiled on motorized winches. Passive pulleys may guide the cables from the winches to the cable exit points. Accordingly, the motion of the moving platform is controlled by modifying the cable lengths. CDPRs have several advantages such as a relatively low mass of moving parts and a potential large workspace. As a consequence, they can be used in several applications such as heavy payload handling and airplane painting [1], large-scale assembly and handling operations [2], and fast pickand-place operations [3]. Other possible applications include the broadcasting of sporting events, haptic devices [4] and search and rescue deployable platforms [5]. Some recent works have dealt with the design and reconfiguration planning of reconfigurable CDPRs that can be used in cluttered industrial environments for painting and sandblasting large structures [6]. It should be noted that redundant actuated CDPRs are more appropriate than cranes for accurate pick-and-place operations and large and heavy parts because the former suffer less from payload swinging than the latter. Moreover, CDPRs can control both the position and the orientation of the object in contrary to classical cranes. Accordingly, this paper deals the determination of a control solution for a suspended CDPR semi-industrial prototype with highly variable payload.

The control scheme should be robust with respect to the uncertain payload and lead to good moving-platform pose accuracy. CDPR control strategies are usually based on PD controllers [3], which can be completed with feedforward terms to predict the moving-platform dynamic behavior. Some control schemes for CDPRs use adaptive control to estimate parameters that are not well identified, such as friction coefficients and variable parameters like the payload mass [7]. The outputs of these controllers can either be a set of desired cable lengths or cable tensions. Knowledge of cable tensions is interesting to detect cable sagging [8] or to implement a tension distribution strategy [9].

The objective of the experiments presented in this paper is to determine whether the estimation of the payload mass and the addition of a compensating feedforward term can improve the robustness of CDPR control or not. An estimation of the payload mass and center of gravity using dynamometers, force sensors attached between the platform and the cables, was introduced in [10] in a position controller to compensate for cable elasticity, with loads up to $40 \mathrm{~kg}$. In this paper, we use a similar approach to estimate and update the platform mass in real-time in the feedforward term of the a PD torque controller on an industrial robot with heavy payloads, up to a total mass of $615 \mathrm{~kg}$.

The paper is organized as follows. Sec. 2 presents the CDPR semi-industrial prototype used for the experimental tests as well as its modeling. Sec. 3 describes the experimental setup and test trajectory used to compare the three control schemes, which are explained in Secs. 4 and 5. Experimental results are presented and analyzed in Sec. 6. Finally, conclusions are drawn and future work is presented in Sec. 7.

\section{PROTOTYPE DESCRIPTION AND MODELING}

This section deals with a description and modeling of the CDPR prototype, named CAROCA [11], shown in Fig. 1 and used for the experimental comparison of the control schemes.

\subsection{CAROCA prototype and ROCKET project}

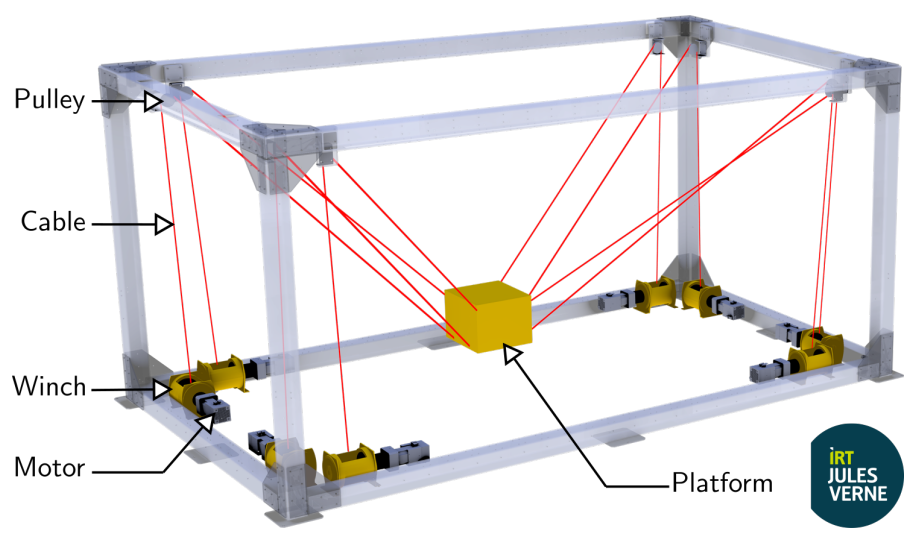

FIGURE 1. CAROCA PROTOTYPE 3D MODEL IN A SUSPENDED CONFIGURATION.

CAROCA is a reconfigurable CDPR prototype developed at IRT Jules Verne in the framework of a project dedicated to industrial operations. The aim of the ROCKET project is the displacement of metal parts of highly variable shapes and mass, up to $700 \mathrm{~kg}$, through the development of a robust control method regarding these aspects. This prototype, is reconfigurable because its pulleys can be displaced in a discrete manner on its frame, allowing the robot to be assembled both in a suspended configuration and in a fully-constrained configuration depending on the targeted application. In this paper, the suspended configuration is considered. The size of the prototype is $7 \mathrm{~m}$ long, $4 \mathrm{~m}$ wide and $3 \mathrm{~m}$ high. It is composed of eight cables coiled around $120 \mathrm{~mm}$ diameter Huchez ${ }^{\mathrm{TM}}$ winches, which are pulling a moving-platform. The winches are actuated by B\&R Automation $^{\mathrm{TM}}$ synchronous motors of nominal speed and nominal torques equal to $2200 \mathrm{rpm}$ and $15.34 \mathrm{Nm}$, respectively. A two-stage gearbox of reduction ratio equal to 40 is assembled between each motor and each winch. As a consequence, the prototype is capable of lifting up to $1 \mathrm{~T}$.

Figure 2 presents the ROCKET moving-platform (MP) of size $1.5 \mathrm{~m} \times 1.5 \mathrm{~m} \times 1 \mathrm{~m}$ and mass $366 \mathrm{~kg}$. Five magnets are embedded under the moving platform to pick metal parts. The robot 
is also equipped with Tractel ${ }^{\mathrm{TM}}$ dynamometers located between the cables and the anchor points of the platform, as shown in Fig. 2. Those dynamometers deliver an intensity between 4 and $20 \mathrm{~mA}$, proportional to the tension in the corresponding cable up to $25000 \mathrm{~N}$, and have been calibrated for the typical working range of the robot comprised between 0 and $5000 \mathrm{~N}$, while using a reference dynamometer. Hardware such as motors and control bay are standard industrial components commercialized by $\mathrm{B} \& \mathrm{R}$ Automation ${ }^{\mathrm{TM}}$. The robot programming is done under Automation Studio $4.1^{\mathrm{TM}}$ and runs in a $10 \mathrm{~ms}$ real-time loop. The control schemes of the robot are developed and simulations are performed with MATLAB ${ }^{\circledR}$ and Simulink ${ }^{\circledR}$.

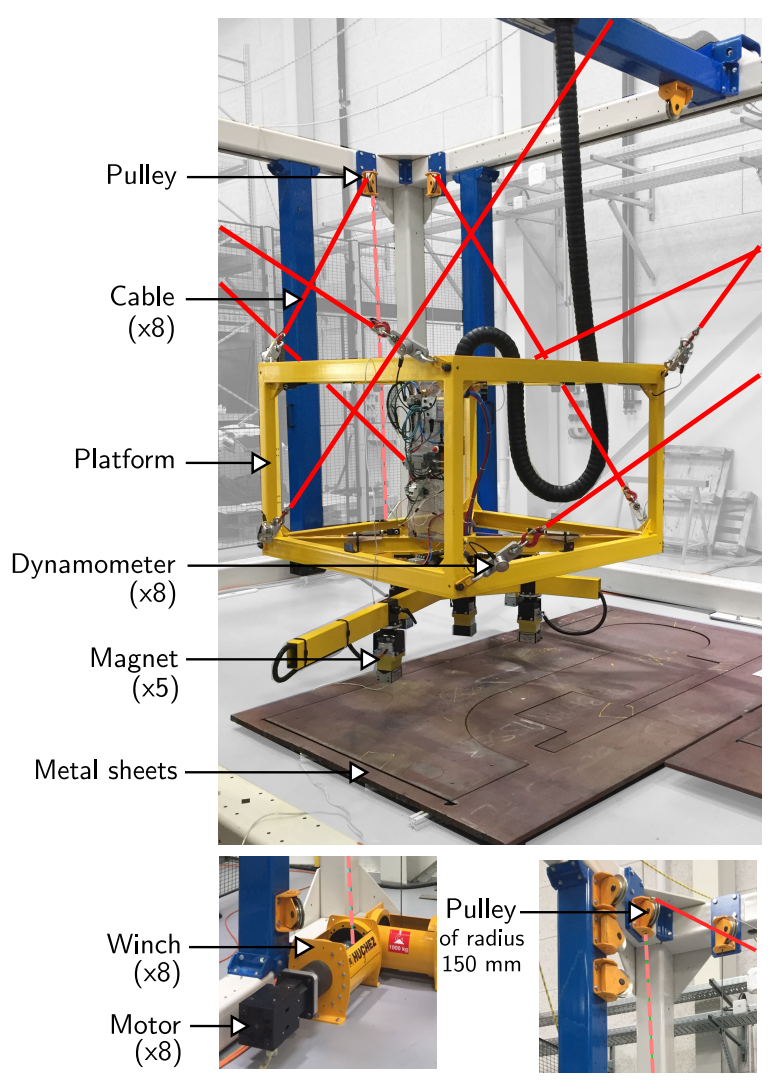

FIGURE 2. TOP. THE MOVING-PLATFORM (MP) EQUIPPED WITH FIVE MAGNETS TO PICK METAL PARTS AND EIGHT DYNAMOMETERS TO MEASURE CABLE TENSIONS. BOTTOM. A MOTOR, A WINCH AND A PULLEY.

\subsection{Modeling}

The geometric, kinematic and dynamic modelings of CAROCA are written thereafter.
2.2.1 Geometric model Figure 3 depicts the main geometric parameters of a CDPR and its $i^{\text {th }}$ loop-closure equation, $i \in\{1, \ldots, m\}, m$ being the number of cables attached to the MP, $\mathscr{F}_{b}$ is the robot base frame, and $\mathscr{F}_{p}$ is the MP frame.

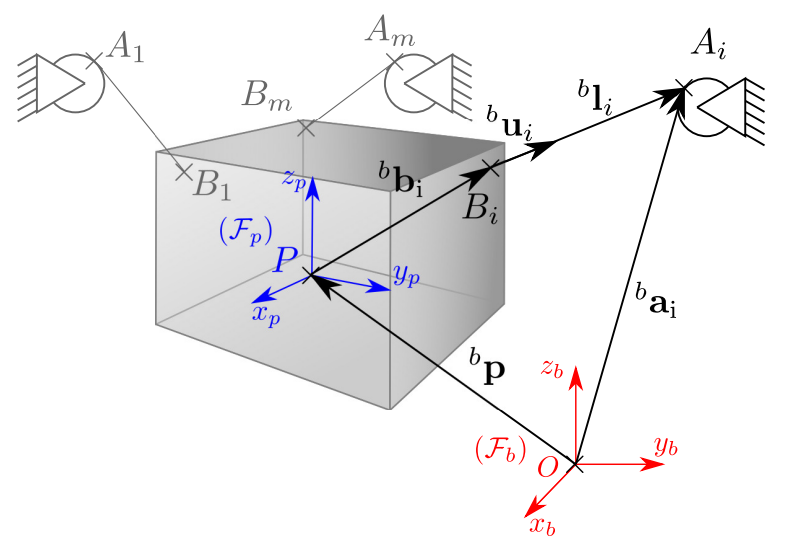

FIGURE 3. CDPR GEOMETRIC PARAMETERIZATION.

Cable exit points are denoted as $A_{i}$, while cable anchor points are denoted as $B_{i}$. Vector ${ }^{b} \mathbf{a}_{i}$ points from point $O$ to point $A_{i}$ and is expressed in frame $\mathscr{F}_{b}$. Vector ${ }^{p} \mathbf{b}_{i}$ point from point $P$ to point $B_{i}$ and is expressed in frame $\mathscr{F}_{p}$. Vector ${ }^{b} \mathbf{p}$ is the position vector of point $P$, the MP geometric center, expressed in $\mathscr{F}_{b}$.

Vector $\mathbf{l}_{i}$ represents the $i^{\text {th }}$ cable vector and points from $B_{i}$ to $A_{i}$, and reads as :

$$
{ }^{b} \mathbf{l}_{i}=l_{i}{ }^{b} \mathbf{u}_{i}={ }^{b} \mathbf{a}_{i}-{ }^{b} \mathbf{p}-{ }^{b} \mathbf{R}_{p}{ }^{p} \mathbf{b}_{\mathbf{i}}
$$

with ${ }^{b} \mathbf{R}_{p}$ the rotation matrix from frame $\mathscr{F}_{b}$ to frame $\mathscr{F}_{p} . l_{i}$ is the length of the $i^{t h}$ cable and $\mathbf{u}_{i}$ is the unit vector of the $i$ th cable vector, defined as

$$
l_{i}=\left\|{ }^{b} \mathbf{l}_{i}\right\|_{2} \quad \quad{ }^{b} \mathbf{u}_{i}=\frac{{ }^{b} \mathbf{l}_{i}}{\left\|{ }^{b} \mathbf{l}_{i}\right\|_{2}}
$$

where $\|\cdot\|_{2}$ denotes the Euclidean norm of a vector.

2.2.2 Static and kinematic models The static equilibrium of the platform is given by

$$
\mathbf{W t}+\mathbf{w}_{e}+\mathbf{w}_{g}=0
$$


with $\mathbf{W}$ named as the wrench matrix of the robot and expressed as

$$
\mathbf{W}=\left[\begin{array}{ccccc}
{ }^{b} \mathbf{u}_{1} & \ldots & { }^{b} \mathbf{u}_{i} & \ldots & { }^{b} \mathbf{u}_{m} \\
{ }^{b} \mathbf{b}_{1} \times{ }^{b} \mathbf{u}_{1} & \ldots & { }^{b} \mathbf{b}_{i} \times{ }^{b} \mathbf{u}_{i} & \ldots & { }^{b} \mathbf{b}_{m} \times{ }^{b} \mathbf{u}_{m}
\end{array}\right]
$$

$\mathbf{t}$ is the cable tension vector. $\mathbf{w}_{g}$ is the wrench applied to the platform due to gravity and $\mathbf{w}_{e}$ is an external wrench expressed in the fixed reference frame $\mathscr{F}_{b}$ and defined as

$$
\mathbf{w}_{e}=\left[\mathbf{f}_{e}^{T} \mathbf{m}_{e}^{T}\right]^{T}=\left[f_{x} f_{y} f_{z} m_{x} m_{y} m_{z}\right]^{T}
$$

where $f_{x}, f_{y}$ and $f_{z}$ are the components of the external force vector $\mathbf{f}_{e}$, and $m_{x}, m_{y}$ and $m_{z}$ are the components of the external moment vector $\mathbf{m}_{e}$. The components of the external wrench $\mathbf{w}_{e}$ are assumed to be bounded.

The kinematic model of the CDPR is defined as

$$
\mathbf{J v}=-\mathbf{i} \quad \text { with } \quad \mathbf{v}=\left[\begin{array}{ll}
{ }^{b} \dot{\mathbf{p}} & { }^{b} \boldsymbol{\omega}
\end{array}\right]^{T}
$$

with $\dot{l}_{i}>0$ corresponding to cable unwinding, ${ }^{b} \omega$ being the angular velocity of the MP expressed in $\mathscr{F}_{b}$ and $\mathbf{J}$ the kinematic Jacobian matrix of the CDPR obtained from $\mathbf{W}$ as:

$$
\mathbf{J}=-\mathbf{W}^{T}
$$

2.2.3 Dynamic model From [11], the dynamic model of the CDPR can be written as

$$
\mathbf{W t}-\mathbb{I}_{p} \dot{\mathbf{v}}-\mathbf{C v}+\mathbf{w}_{e}+\mathbf{w}_{g}=0
$$

with $\mathbb{I}_{p}$ the spatial inertia of the platform and $\mathbf{C}$ the matrix of the centrifugal and Coriolis wrenches. Given that the center of mass of the platform $G$ does not coincide with the origin of $\mathscr{F}_{p}$, the wrench $\mathbf{w}_{g}$ due to the gravity acceleration $\mathbf{g}$ is defined as

$$
\mathbf{w}_{g}=\left[\begin{array}{l}
m_{p} \mathbf{I}_{3} \\
\mathbf{M} \hat{\mathbf{S}}_{p}
\end{array}\right] \mathbf{g}
$$

with $m_{p}$ the mass of the platform, $\mathbf{I}_{3}$ the $3 \times 3$ identity matrix, $\mathbf{M S}_{p}={ }^{b} \mathbf{R}_{p}\left[m_{p} x_{G}, m_{p} y_{G}, m_{p} z_{G}\right]^{\mathrm{T}}$ the first momentum of the moving platform defined with respect to frame $\mathscr{F}_{b}$. The vector $\mathbf{S}_{p}=\left[x_{G}, y_{G}, z_{G}\right]^{\mathrm{T}}$ defines the position of $G$ in $\mathscr{F}_{p} . \mathbf{M} \hat{\mathbf{S}}_{p}$ is the skew-symmetric matrix associated to $\mathbf{M S}_{p}$.

$\mathbb{I}_{p}$ represents the spatial inertia of the platform, and reads as

$$
\mathbb{I}_{p}=\left[\begin{array}{cc}
m_{p} \mathbf{I}_{3} & -\mathbf{M} \hat{\mathbf{S}}_{p} \\
\mathbf{M} \hat{\mathbf{S}}_{p} & \mathbf{I}_{p}
\end{array}\right]
$$

with $\mathbf{I}_{p}$ the inertia tensor matrix of the platform, which can be computed from the platform's inertia tensor $\mathbf{I}_{g}$, using the Huygens-Steiner theorem

$$
\mathbf{I}_{p}={ }^{b} \mathbf{R}_{p} \mathbf{I}_{g}{ }^{b} \mathbf{R}_{p}^{\mathrm{T}}-\frac{\mathbf{M} \hat{\mathbf{S}}_{p} \mathbf{M} \hat{\mathbf{S}}_{p}}{m_{p}}
$$

$\mathbf{C}$ is the matrix of the centrifugal and Coriolis wrenches with

$$
\mathbf{C v}=\left[\begin{array}{c}
{ }^{b} \hat{\omega}^{b} \hat{\omega} \mathbf{M} \mathbf{S}_{p} \\
{ }^{b} \hat{\omega} \mathbf{I}_{p}{ }^{b} \omega
\end{array}\right]
$$

where ${ }^{b} \hat{\omega}$ the skew-symmetric matrix associated to ${ }^{b} \omega$.

\section{TEST TRAJECTORY AND EXPERIMENTAL SETUP}

The objective of the experiments presented in this paper is to determine if the payload mass estimation can improve the robustness of CDPR control and to quantify the performance improvement. In this section, the test trajectory and the controllers architectures used in the sequel are defined. A video of the experiments along the trajectory is available ${ }^{1}$.

\subsection{Test trajectory}

In order to evaluate the performances of different control methods, a desired trajectory describing a typical pick-and-place application has been generated (see Figs. 4-5). We define the $\mathrm{x}$-axis of the frame $\mathscr{F}_{b}$ along the width of the CDPR, the y-axis along its length and the z-axis along its height. The trajectory consists of:

1. AB: $200 \mathrm{~mm}$ vertical displacement up;

2. BC: arc along the diagonal of the base footprint, with simultaneous displacements of $300 \mathrm{~mm}$ up, $300 \mathrm{~mm}$ along the $\mathrm{x}$-axis and $1400 \mathrm{~mm}$ along the $\mathrm{y}$-axis;

3. CD: arc along the diagonal of the base footprint, with simultaneous displacements of $300 \mathrm{~mm}$ down, $300 \mathrm{~mm}$ along the $\mathrm{x}$-axis and $1400 \mathrm{~mm}$ along the $\mathrm{y}$-axis;

4. DE: $200 \mathrm{~mm}$ vertical displacement down;

5. EA: back to point A while following the same path. 

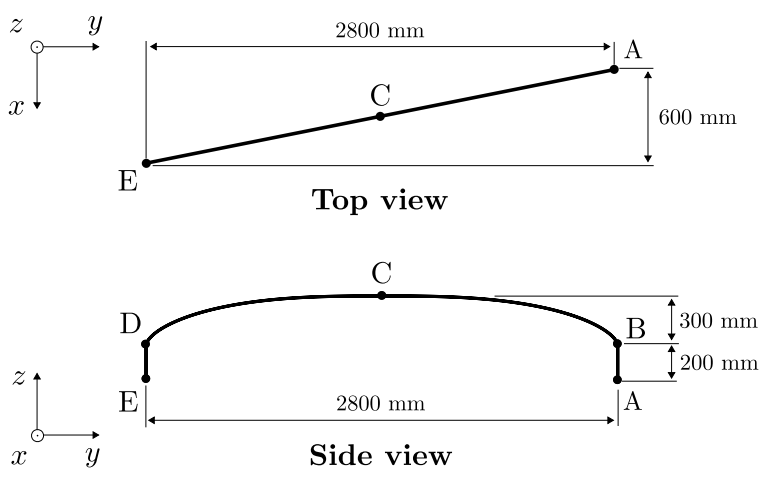

FIGURE 4. TOP AND SIDE VIEWS OF THE TEST TRAJECTORY.

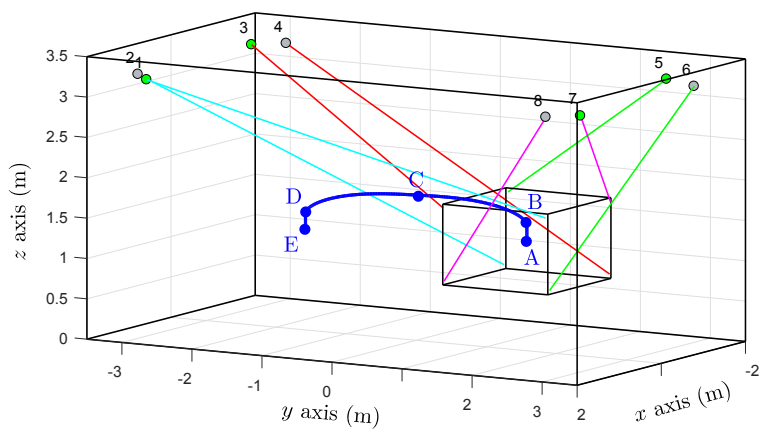

FIGURE 5. TEST TRAJECTORY IN BLUE AND CDPR CONFIGURATION.

The platform moves from $\mathrm{A}$ to $\mathrm{E}$ in $30 \mathrm{~s}$, and from $\mathrm{E}$ to $\mathrm{A}$ in $30 \mathrm{~s}$.

The trajectory is generated using s-curves, which ensure continuous velocity and acceleration trajectory profiles as explained in [12]. This test is first performed on the CDPR with the empty platform. In order to evaluate the control robustness, the platform of mass $366 \mathrm{~kg}$ is then loaded with either:

a plate $\mathrm{M}_{1}$ of mass $122 \mathrm{~kg}$ for a total load weighed $488 \mathrm{~kg}$ a plate $\mathrm{M}_{2}$ of mass $249 \mathrm{~kg}$ for a total load weighed $615 \mathrm{~kg}$

Note that the mass variation is significant, namely $+33 \%$ and $+68 \%$ with respect to the MP mass, respectively. The low accelerations along the test trajectory are desirable for the payload stability.

\subsection{Available measurements}

Angular position, velocity and torque are available for each motor. However, estimation of the cable tensions from the motor torques is affected by losses due to frictions in motors, gearbox, winches and pulleys [10]. Moreover, torque measure issued from the motors currents is often imprecise. For these reasons, we use dynamometers to obtain a direct measurement of the eight cable tensions.

\footnotetext{
${ }^{1}$ Experiments video : http://bit.ly/DETC2018-85304
}

While a direct measurement of the robot position is not possible in the absence of external sensors, it is possible to estimate the platform position by solving a set of non-linear equations [13] with a Least Square Method with lsqnonlin in MATLAB for example, defined by:

$$
\mathbf{X}^{*} \rightarrow \min _{\mathbf{p}^{*}, \phi^{*}} \sum_{i=1}^{m}\left(l_{i}-\hat{l}_{i}(\mathbf{p}, \phi)\right)^{2}
$$

where $m$ is the total number of cables, $l_{i}$ the real $i^{\text {th }}$ cable length, $\hat{l}_{i}$ an estimated $i^{\text {th }}$ cable length for a platform position $\mathbf{p}^{*}$ and orientation $\phi^{*}$. At each time step, a numerical solution to the direct geometric model is a pose $\mathbf{X}^{*}=\left[\mathbf{p}^{*} \phi^{*}\right]$ for which the difference between $l_{i}$ and $\hat{l}_{i}$ is inferior to the desired error. Because of the computing cost of solving these equations, we only use this information to analyze the results presented in Sec. 6.

\subsection{Control strategies}

The test trajectory described in Section 3.1 gives the desired MP pose, i.e. MP position and orientation, which are converted into desired motor positions and velocities using the inverse CDPR geometric and kinematic models, from Equations (1) and (6). Because of the parallel nature of CDPRs, all signals are treated as vectors in the following control schemes. Each actuation chain, from the motors to the cables, has its own controller that takes desired and measured motor position and velocity as input. The output is the corresponding desired motor torque. Here, the eight decentralized controllers were tuned in similar manner. A linear friction model was implemented in each actuation chain to compensate the losses in the transmissions:

$$
\tau_{f}=\mathbf{F}_{c} \operatorname{sign}(\dot{\mathbf{q}})+\mathbf{F}_{v} \dot{\mathbf{q}}
$$

with $\tau_{f}$ the generated friction compensation and $\dot{\mathbf{q}}$ is the measured motor rate vector. $\mathbf{F}_{c}$ and $\mathbf{F}_{v}$ are respectively the vectors containing the static and viscous friction coefficients separately identified for each motor.

In what remains, two common CDPR control strategies are tested: a PD controller and a PD controller with feedforward [3], [14]. A novel controller based on a real-time estimation of the payload mass from dynamometers measurements is then developed. Eventually, its performance are compared to those of the first two controllers.

\section{PROPORTIONAL-DERIVATIVE CONTROLLER}

The first control scheme implemented on this robot is based on a proportional-derivative strategy that is well established in 
robotics including CDPRs [3]. The control scheme is presented in Fig. 6, with $\mathbf{X}_{d}$ the 6-dimensional vector containing the desired Cartesian position and orientation of MP, $\dot{\mathbf{X}}_{d}$ the desired MP twist, $\mathbf{q}_{d}$ the 8-dimensional vector of desired angular positions of the motors, $\tau_{m}$ the 8-dimensional vector of desired motors torques. $K_{p}$ and $K_{d}$ are respectively the proportional and derivative gains of the controller, tuned so as the robot achieves accuracy and stability with the MP alone. The same values were used in the two other controllers.

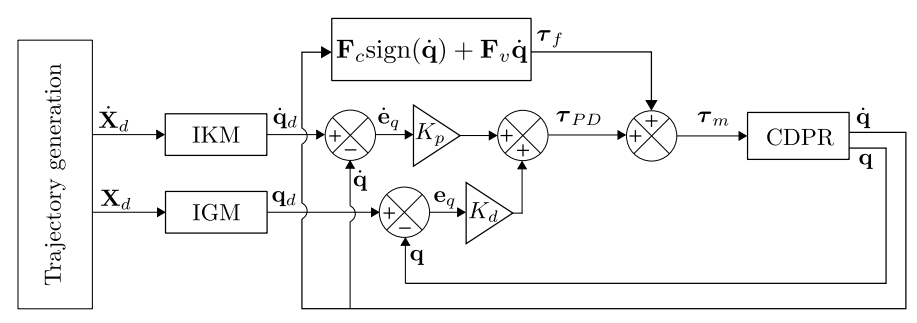

FIGURE 6. PD CONTROL SCHEME FOR THE CDPR.

\section{MASS COMPENSATION}

While a PD controller is the basis of robot control, an feedforward term is commonly included in CDPR control strategies $[7,15]$ to predict the dynamics of the platform and improve the accuracy of the robot.

\subsection{PD controller with MP mass compensation}

Based on the dynamic model of the robot, it is possible to add a term to the controller that will anticipate for the MP dynamics and compensate for a given mass along the trajectory. From Eqn. (8), this feedforward term is defined as

$$
\tau_{F F}=\frac{m_{p} R \mathbf{W}^{+}\left[\ddot{\mathbf{X}}_{d}+\mathbf{g}\right]}{r}
$$

with $\tau_{F F}$ the feedforward torque, $m_{p}$ the mass to compensate composed of the platform and its eventual load, $g$ the vector representing gravity in the base frame, $R$ the radius of the winches and $r$ the gearbox reduction. In this particular controller, $m_{p}$ equals to $m_{M P}$ the mass of the MP only as the real payload remains unknown. $\mathbf{W}^{+}$denotes the Moore-Penrose pseudo-inverse of $\mathbf{W}$. Figure 7 presents the control scheme of the PD controller with feedforward (PDFF), where $\ddot{X}_{d}$ contains the Cartesian acceleration and angular acceleration of the platform.

\subsection{Real-time mass estimation}

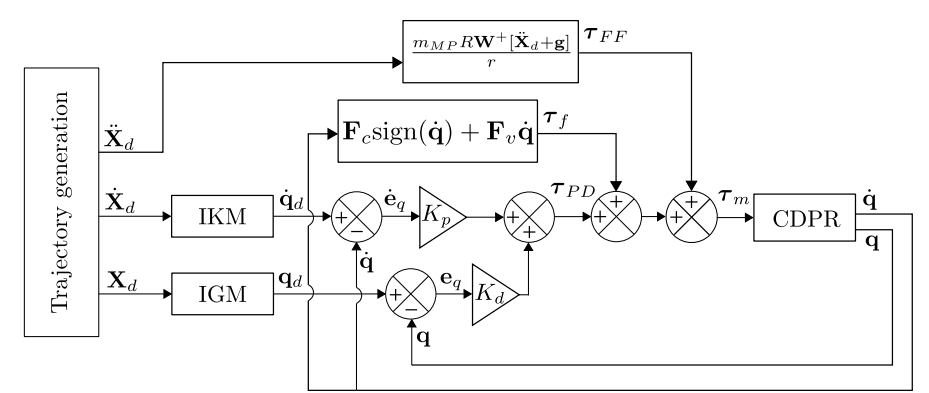

FIGURE 7. CONTROL SCHEME OF A CDPR PD CONTROLLER AUGMENTED WITH FEEDFORWARD TERM (PDFF).

In order to improve robustness, it is interesting to update the mass in the compensation term, especially if in the considered applications, objects have highly various shapes, sizes and weights. With the following assumptions:

1. the linear and angular velocity of the platform are low

2. the only wrench applied to the platform is due to the carried payload, namely, the metal plate

3. the cables are straight and inelastic

the inertial and Coriolis effect on the platform can be neglected and Eqn. (8) can be rewritten as:

$$
\mathbf{W t}+\mathbf{w}_{g}=0
$$

Knowing the wrench matrix $\mathbf{W}$, the cable tension vector $\mathbf{t}$ measured by the dynamometers and $\mathbf{w}_{g}$ expressed in Eqn. (9), it is then possible to calculate the payload mass $m_{p}$ and the Cartesian coordinates $x_{G}$ and $y_{G}$ of the center of mass of the set composed of the moving-platform and the carried payload [10], expressed in $\mathscr{F}_{p}$. Assuming a null platform orientation along the test trajectory, Eqn. (16) becomes

$$
\mathbf{W}\left[\begin{array}{c}
t_{1} \\
\vdots \\
t_{i} \\
\vdots \\
t_{8}
\end{array}\right]+\left[\begin{array}{c}
0 \\
0 \\
-m_{p} g \\
-m_{p} g y_{G} \\
m_{p} g x_{G} \\
0
\end{array}\right]=0
$$

Because of the complexity of the direct geometric model and in absence of external sensors, no information on the actual pose of the platform is available. For this reason, the desired positions of the platform from the trajectory generation are used to calculate the wrench matrix $\mathbf{W}$ along the trajectory.

Figure 8 and presents the estimated masses for the three payloads along the test trajectory using the proposed method. The 
estimations are stable at constant velocity of the MP. Errors during the $\mathrm{x}-\mathrm{y}$ translation phase can be explained by differences between the real robot and its model, as cable exit points $A_{i}$ are assumed fixed in space but can move on the $150 \mathrm{~mm}$ radius of CAROCA 2-DoF pulleys. This introduces differences in cable orientations and leads to slightly underestimated masses. The estimations variations during vertical motions, from $0 \mathrm{~s}$ to $5 \mathrm{~s}, 25 \mathrm{~s}$ to $35 \mathrm{~s}$ and $55 \mathrm{~s}$ to $60 \mathrm{~s}$, are due to the platform vibrations. The mean of the mass estimation and the standard deviation (STD) is provided for the three payloads in Tab. 1; the relative errors are below $5 \%$ in each scenario. These results motivate the consideration of the mass estimation in a control strategy.

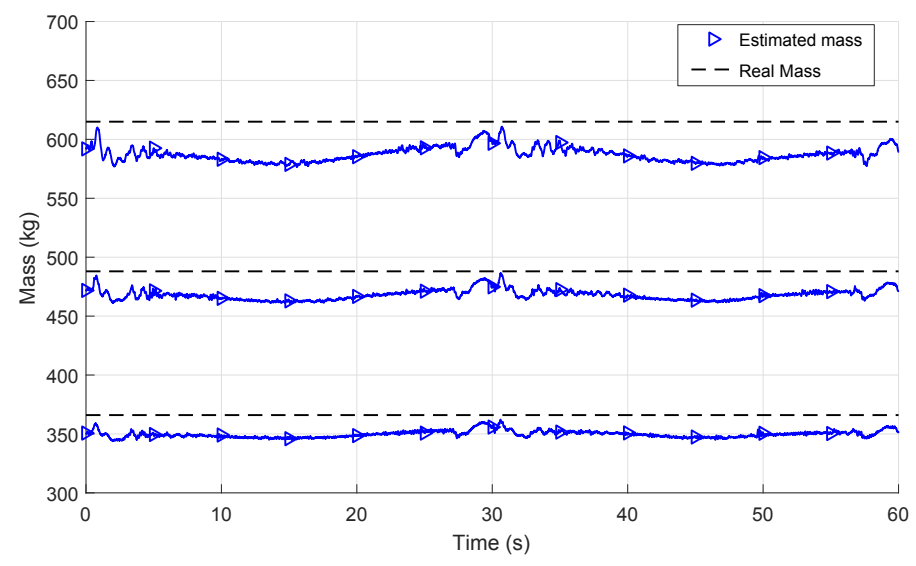

FIGURE 8. ESTIMATED MASS (KG) ALONG THE TEST TRAJECTORY FOR THE THREE PAYLOADS.

TABLE 1. MEAN ESTIMATED MASS ALONG TEST TRAJECTORY FOR THE THREE PAYLOADS.

\begin{tabular}{ccccc}
\hline \hline Payload & Theoretical & Estimation & Error & STD \\
\hline $\mathrm{MP}$ & $366 \mathrm{~kg}$ & $346.8 \mathrm{~kg}$ & $5 \%$ & $2.8 \mathrm{~kg}$ \\
\hline $\mathrm{MP}+\mathrm{M}_{1}$ & $488 \mathrm{~kg}$ & $466.3 \mathrm{~kg}$ & $4 \%$ & $4.3 \mathrm{~kg}$ \\
\hline $\mathrm{MP}+\mathrm{M}_{2}$ & $615 \mathrm{~kg}$ & $587.0 \mathrm{~kg}$ & $5 \%$ & $6.2 \mathrm{~kg}$ \\
\hline \hline
\end{tabular}

Although the pick and place trajectory does not contain orientations of the MP, the stability of the estimation was tested for rotations around the three axis, as illustrated in Fig. 9. Figure 10 presents the mass estimation while the platform, placed in the center of the workspace, was successively rotated of 15 degrees around $x, y$ then $z$. At $27 \mathrm{~s}$, the platform was rotated of 10 degrees along the three axis simultaneously. Even if variations are visible at the start and end of each motion, due to vibrations in the platform and cables, it is visible that the mass estimation remained stable during these orientations, with a standard deviation of only $1.1 \mathrm{~kg}$ during this experiment.

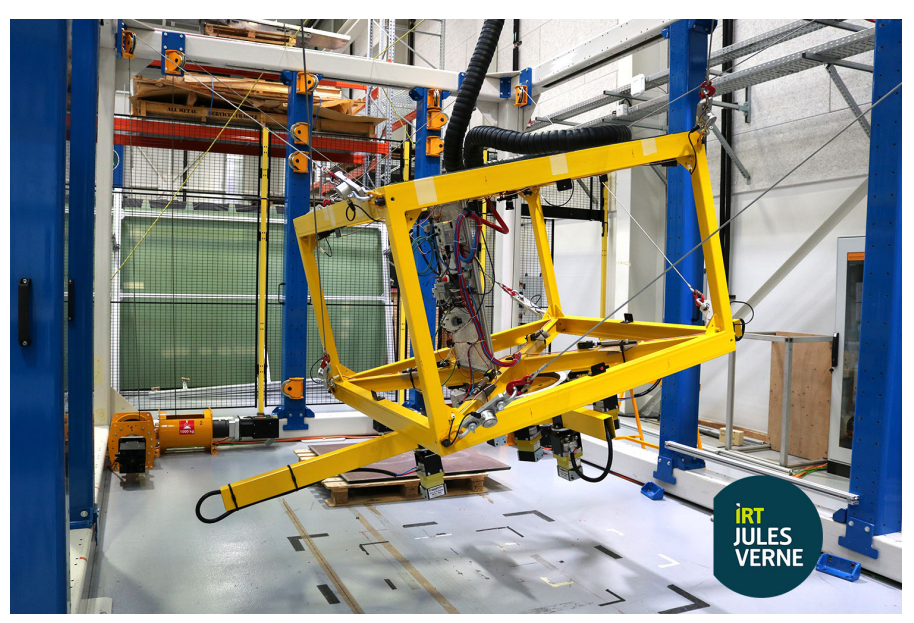

FIGURE 9. MP ROTATED OF 15 DEGREES AROUND Y AXIS

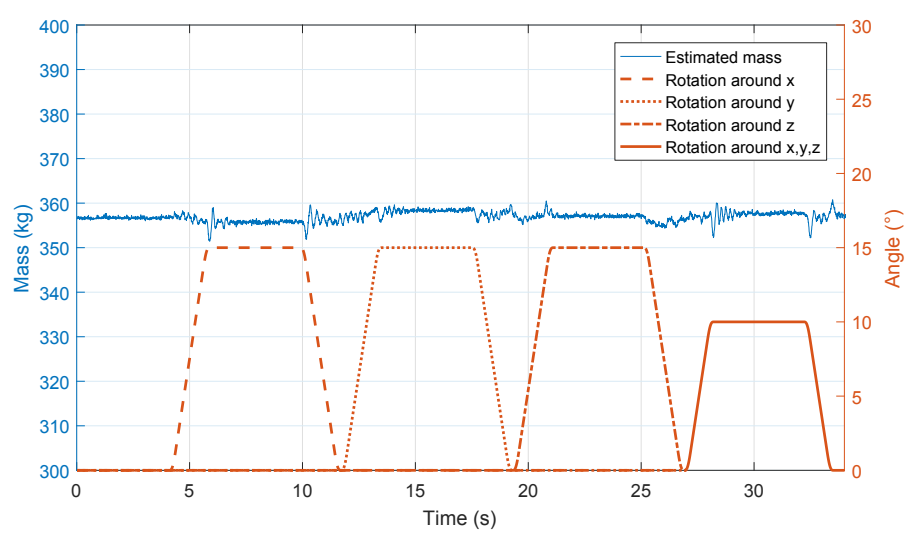

FIGURE 10. ESTIMATED MASS (KG) OF MP DURING ORIENTATIONS

The control method should be robust with regard to fast payload changes during pick and place operations. Accordingly, the CDPR was loaded with the $\mathrm{MP}+\mathrm{M}_{2}$ and the mass $\mathrm{M}_{2}$ was suddenly dropped during the tests. The recorded mass estimation is shown in Fig. 11. Oscillations can be observed as the platform oscillates once $M_{2}$ is released. This effect can be attributed to the cable elasticity, which is an ongoing subject of research [16] for CDPR. It can be noted that the oscillations are naturally damped in less than two seconds. It will be relevant to consider cable 
elasticity in future work to further improve the robustness of the proposed control method.

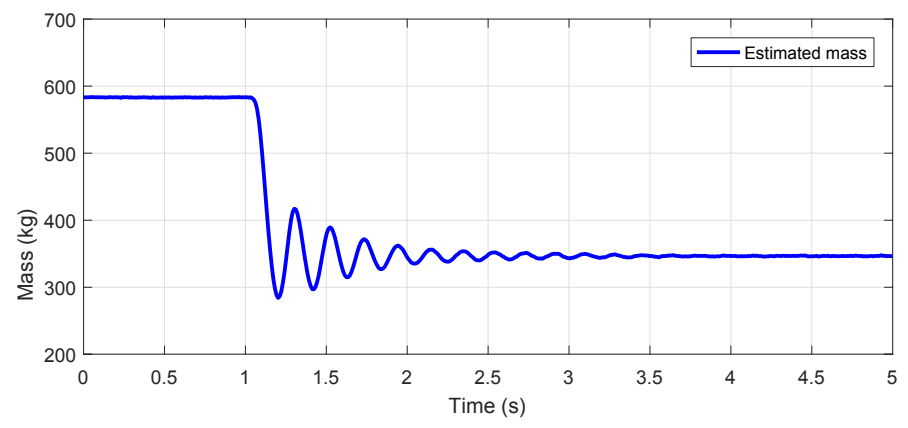

FIGURE 11. STARTING WITH PAYLOAD MP+M $\mathrm{M}_{2}$, ESTIMATED MASS (KG) DURING A SUDDEN DROP OF $\mathrm{M}_{2}$

\subsection{PD controller with real-time mass estimation and compensation}

From the evaluation of the payload mass a new controller is designed, which updates the value of the mass in the feedforward term expressed in Eqn. (15) in order to adapt the controller to a new payload. This time $m_{p}$ describes the estimated total payload, namely the MP and a possible metal plate. The control scheme of this PD torque controller with real-time update of the feedforward term (PDFFRT) is depicted in Fig. 12.

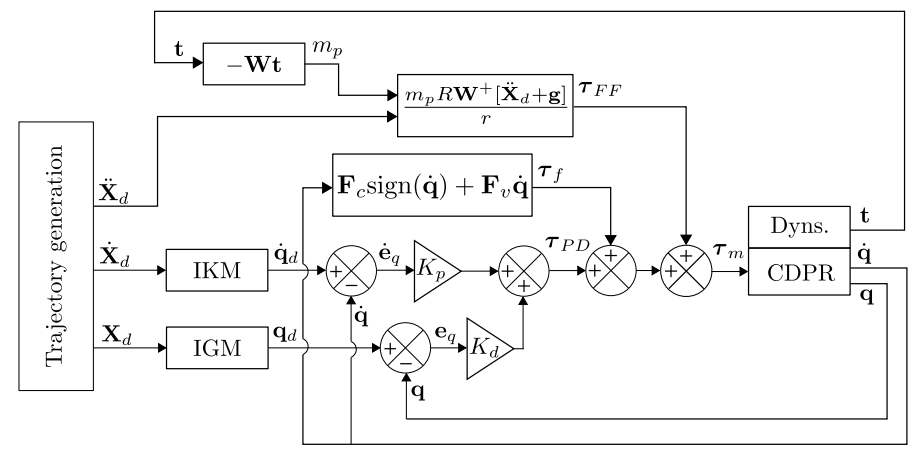

FIGURE 12. CONTROL SCHEME WITH FEEDFORWARD FOR REAL-TIME MASS ESTIMATION AND COMPENSATION (PDFFRT).

\section{EXPERIMENTAL RESULTS}

Experiments have been realized on the CAROCA prototype along the test trajectory with the three controllers and their per- formance are compared.

\subsection{Cartesian errors}

Although we do not have external sensors to obtain the MP actual pose, errors in position and orientation in the task space of the robot were approximated by solving the direct geometric model as shown in Eqn. 13. The errors in Cartesian position of the MP with the different controllers and payloads along the $\mathrm{z}$ axis are presented in Fig. 13. As the impact of the payload mass is directed along the gravity, we focus on the platform position accuracy along the $\mathrm{z}$-axis. We noted that the $\mathrm{x}$-axis and $\mathrm{y}$-axis precision were only slightly impacted by the payload mass increment with a maximum error of less than $10 \mathrm{~mm}$, although we noted a small improvement with the addition of the feedforward term, down to a $5 \mathrm{~mm}$ error.

It is apparent that the PDFFRT leads the smallest errors from the three controllers. The PD controller suffers from an immediate error at the beginning of the trajectory, as the initial output torque of the PD is null and the platform position slightly drops under load. Between the lightest and the heaviest payloads, the errors of both the PD and PDFF controllers have increased by more than $20 \mathrm{~mm}$. On the other hand, the PDFFRT controller maintains a low error, smaller than $5 \mathrm{~mm}$, for each payload. Although there are still some variations in the position accuracy, this behavior is stable enough to pick the heaviest objects without too much precision loss.

While the performance of the PD controller and PD controller with nominal mass compensation are affected by the mass increment, the real-time mass estimation allows to keep a more precise control of the platform position.
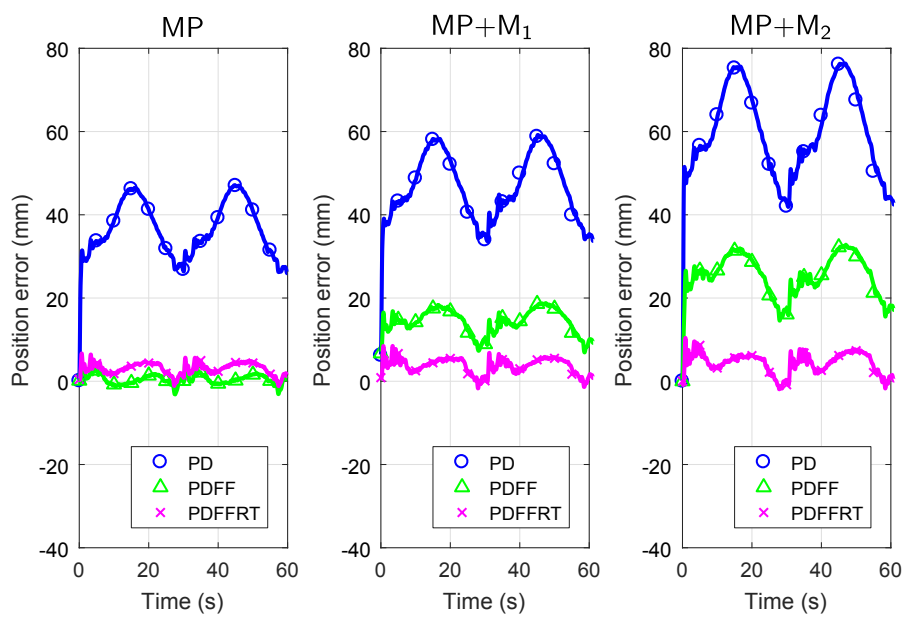

FIGURE 13. PLATFORM POSITION ERROR ALONG Z-AXIS (MM) VERSUS TIME (S) OBTAINED WITH THE THREE CONTROLLERS, FOR SEVERAL MASSES. LEFT. MP (366 KG). CENTER. MP+M $1488 \mathrm{KG})$. RIGHT. $\mathrm{MP}+\mathrm{M}_{2}(615 \mathrm{KG})$. 


\subsection{Error analysis}

Figure 14 shows the root mean square (RMS), maximum (MAX) and standard deviation (STD) of the Cartesian position error of the platform along the z-axis during the test trajectory defined in Sec. 3.1, with the three controllers and payloads. We can observe that the heavier the payload, the larger the value of the three criteria. This error dramatically increases in the cases of the PD and PDFF controllers, while the real-time mass estimation keeps the errors at a consistently low value. The real-time estimation improves the control robustness with respect to the mass increase compared to the two other strategies.

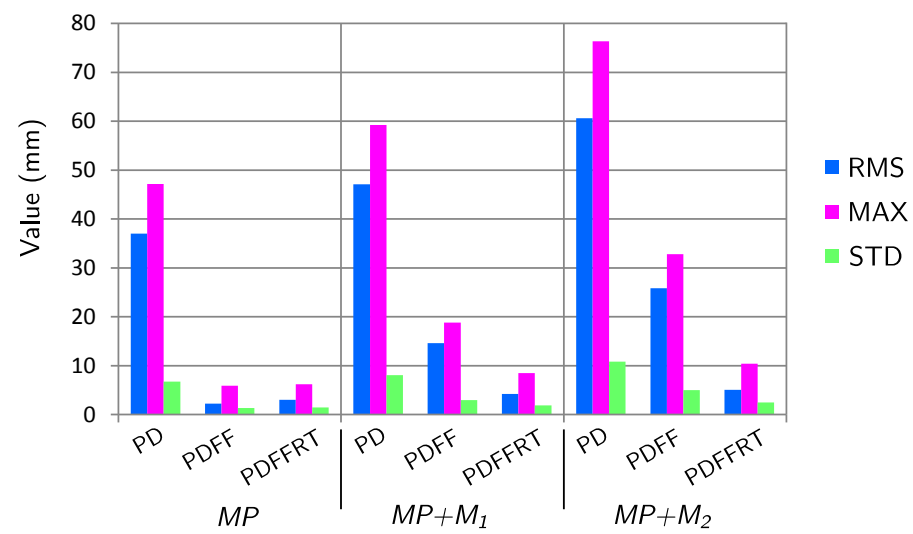

FIGURE 14. RMS, MAXIMUM AND STD OF MP CARTESIAN POSITION ERROR ALONG Z-AXIS FOR THE THREE CONTROLLERS LEFT. PAYLOAD MP CENTER. PAYLOAD MP+M RIGHT. PAYLOAD MP+M 2 .

Figure 15 presents the experimental comparison on the same criteria between the PDFFRT and an ideal $\mathrm{PDFF}^{+}$controller where the mass of the total payload is perfectly known. Here the mass $m_{p}$ given to the feedforward term in Eqn. (15) respectively equals $m_{M P}, m_{M P+M_{1}}$ and $m_{M P+M_{2}}$. We can see from Fig. 15 that the performances of the PDFFRT controller are very close to those of the controller where the mass is known beforehand, which confirms the correctness of the real-time estimation and the robustness of the new controller. In a real application, the ideal $\mathrm{PDFF}^{+}$controller would only be possible if the mass of each part was known and updated at the correct time during the trajectory, which are significant constraints.

\subsection{Contribution of the feedforward and feedback terms}

To complete this analysis, it is interesting to study the contribution of both the proportional-derivative term of the control law, $\tau_{P D}$, and the feedforward term, $\tau_{F F}$, on the control effort. Figure 16 shows the values of those two torques for

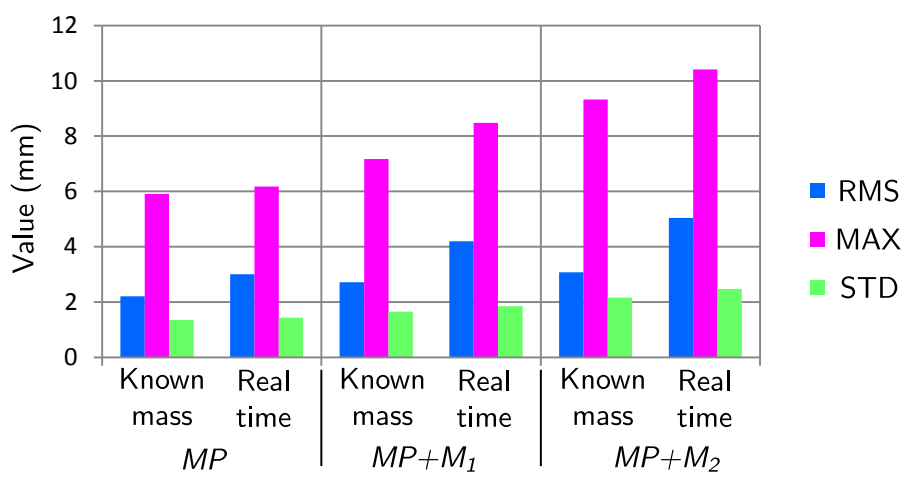

FIGURE 15. RMS, MAXIMUM AND STD OF MP CARTESIAN POSITION ERROR ALONG Z-AXIS FOR THE PDFF WITH KNOWN MASS AND THE PDFFRT CONTROLLERS LEFT. PAYLOAD MP CENTER. PAYLOAD MP+M $\mathrm{M}_{1}$ RIGHT. PAYLOAD $\mathrm{MP}+\mathrm{M}_{2}$.

motor 1 along the test trajectory for the three controllers, while considering the heaviest payload $\left(\mathrm{MP}+\mathrm{M}_{2}\right)$. Torque has a negative value when winding the cables. As the mass estimation is the closest to the real mass of the payload, the feedforward term generates a larger part of the output of the control scheme. For the PDFFRT controller, the feedforward term generates the main part of the required torque to move the platform. However, the feedback part is still necessary to compensate for small errors, due to friction or a shift in the center of mass when picking an object for example.
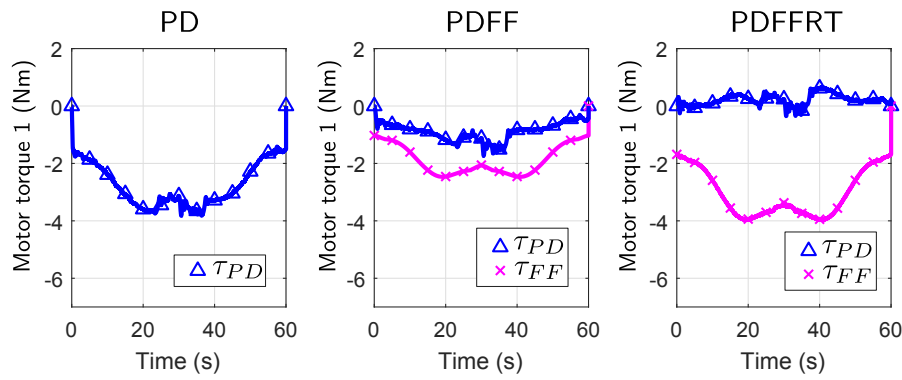

FIGURE 16. TORQUE CONTRIBUTION FROM PD OUTPUT $\left(\tau_{P D}\right)$ AND FEEDFORWARD TERM $\left(\tau_{F F}\right)$ WITH MASS MP+M FOR MOTOR 1 LEFT. PD CONTROLLER CENTER. PDFF CONTROLLER RIGHT. PDFFRT CONTROLLER.

\section{CONCLUSION}

A new control strategy for cable-driven parallel robots (CDPRS) based on a PD torque controller with a real-time mass esti- 
mation and compensation term has been designed, implemented and experimented on a test pick-and-place trajectory with different masses. Using the mass estimation from the dynamometer measurements, it has been possible to continuously update the feedforward term to compensate for any payload and achieve similar performance as with a PD controller with feedforward and exact knowledge of the mass. In particular, the RMS of the platform position error, equal to $60 \mathrm{~mm}$ for the PD controller with the heaviest payload, was reduced by $58 \%$ with the PD controller with feedforward (PDFF), and by $91 \%$ with the controller using real-time mass estimation (PDFFRT). While the PD controller could be specifically tuned for each case, the proposed controller can adapt to the payload, thus improving the robot accuracy.

Later on, the stability of the proposed control schemes will be analyzed. Moreover, a sensitivity analysis will be performed in order to determine the best tuning parameters of the controllers. Further tests will be made to analyze the robustness of the method with regard to high moving-platform accelerations. The mass estimation could also be improved with a finer model of the robot while taking into account the motion of cable exit points due to the pulleys. Finally, the behavior of the robot with regard to payload mass changes during the trajectory will be studied in a future work.

\section{ACKNOWLEDGMENT}

The authors wish to associate the industrial and academic partners of this project, namely, STX France, Clemessy, B\&R Automation, Clarté and CNRS. This work is supported by IRT Jules Verne (French Institute in Research and Technology in Advanced Manufacturing Technologies for Composite, Metallic and Hybrid Structures) in the framework of ROCKET project.

\section{REFERENCES}

[1] Albus, J., Bostelman, R., and Dagalakis, N., 1992. "The NIST SPIDER, A robot crane". Journal of Research of the National Institute of Standards and Technology, 97(3), p. 373.

[2] Pott, A., Meyer, C., and Verl, A., 2010. "Large-scale assembly of solar power plants with parallel cable robots". Isr/Robotik 2010, pp. 999-1004.

[3] Kawamura, S., Kino, H., and Won, C., 2000. "High-speed manipulation by using parallel wire-driven robots". Robotica, 18(1), p. S0263574799002477.

[4] Fortin-Coté, A., Cardou, P., and Gosselin, C., 2014. "An admittance control scheme for haptic interfaces based on cable-driven parallel mechanisms". In Proceedings - IEEE International Conference on Robotics and Automation, pp. 819-825.
[5] Merlet, J. P., and Daney, D., 2010. "A portable, modular parallel wire crane for rescue operations". In Proceedings - IEEE International Conference on Robotics and Automation, pp. 2834-2839.

[6] Gagliardini, L., Caro, S., Gouttefarde, M., and Girin, A., 2016. "Discrete reconfiguration planning for Cable-Driven Parallel Robots". Mechanism and Machine Theory, 100, pp. 313-337.

[7] Lamaury, J., Gouttefarde, M., Chemori, A., and Herve, P. E., 2013. "Dual-space adaptive control of redundantly actuated cable-driven parallel robots". IEEE International Conference on Intelligent Robots and Systems, pp. 48794886.

[8] Meunier, G., Boulet, B., and Nahon, M., 2009. "Control of an overactuated cable-driven parallel mechanism for a radio telescope application". IEEE Transactions on Control Systems Technology, 17(5), pp. 1043-1054.

[9] Gouttefarde, M., Lamaury, J., Reichert, C., and Bruckmann, T., 2015. "A Versatile Tension Distribution Algorithm for 6-DOF Parallel Robots Driven by $\mathrm{n}+2$ Cables". IEEE Transactions on Robotics, 31(6), pp. 1444-1457.

[10] Kraus, W., Schmidt, V., Rajendra, P., and Pott, A., 2013. "Load identification and compensation for a Cable-Driven parallel robot". In Proceedings - IEEE International Conference on Robotics and Automation, pp. 2485-2490.

[11] Gagliardini, L., Gouttefarde, M., and Caro, S. "Determination of a Dynamic Feasible Workspace for Cable-Driven Parallel Robots". pp. 1-10.

[12] Germain, C., Caro, S., Briot, S., and Wenger, P., 2013. "Optimal Design of the IRSBot-2 Based on an Optimized Test Trajectory". In Volume 6A: 37th Mechanisms and Robotics Conference, p. V06AT07A056.

[13] Schenk, C., Masone, C., Pott, A., and Bülthoff, H. H., 2018. "Application of a differentiator-based adaptive supertwisting controller for a redundant cable-driven parallel robot". In Mechanisms and Machine Science, Vol. 53, pp. 254-267.

[14] Lamaury, J., and Gouttefarde, M., 2013. "Control of a large redundantly actuated cable-suspended parallel robot". Proceedings - IEEE International Conference on Robotics and Automation, pp. 4659-4664.

[15] Vafaei, A., Aref, M. M., and Taghirad, H. D., 2010. "Integrated controller for an over-constrained cable driven parallel manipulator: KNTU CDRPM". In Proceedings IEEE International Conference on Robotics and Automation, pp. 650-655.

[16] Baklouti, S., Courteille, E., Caro, S., and Dkhil, M., 2017. "Dynamic and Oscillatory Motions of Cable-Driven Parallel Robots Based on a Nonlinear Cable Tension Model". Journal of Mechanisms and Robotics, 9(6), p. 061014. 